
\title{
28 Research Square \\ Fabrication of High-Entropy Disilicide Nanopowders via Molten Salt-Assisted Magnesium Thermal Reduction
}

Honghua Liu

South China University of Technology

Shanshan Ning

South China University of Technology

B. Du ( $\boldsymbol{\sim}$ dubin@scut.edu.cn )

South China University of Technology https://orcid.org/0000-0002-5242-7378

\section{Rapid Communication}

Keywords: high-entropy ceramics, disilicides, molten salt synthesis, powders

Posted Date: June 19th, 2020

DOI: https://doi.org/10.21203/rs.3.rs-36425/v1

License: (c) (i) This work is licensed under a Creative Commons Attribution 4.0 International License.

Read Full License 


\section{Abstract}

In this work, (Nb 0.25 Ta 0.25 Ti 0.25 Hf 0.25)Si 2 high-entropy disilicide nanopowders were successfully fabricated via molten salt-assisted magnesium thermal reduction for the first time. The results showed that the as-obtained nanopowders possessed a single hexagonal structure (TaSi 2 -type) and consisted of numerous nanopowders with an average particle size of $55 \mathrm{~nm}$. These nanopowders exhibited highly compositional uniformity at microscale, but shown the aggregation of $\mathrm{Ti}$ element at nanoscale. In addition, a typical template formation mechanism was proposed and analyzed in detailed. This work would provide a new way to synthesis of high-entropy disilicise powders for potential high-temperature harsh environment applications.

\section{Introduction}

The newly developed field of crystalline high-entropy ceramics (HECs) are completely disorder solidsolution that consists of four or more components in equiatomic or near equiatomic rations. ${ }^{1,2}$ Compared to conventional single phase ceramics, HECs process unique properties and potential applications in many fields due to their high-entropy, severe lattice-distortion, sluggish diffusion, and cocktail effects. ${ }^{3}$ Heretofore, numerous HECs, including oxides, ${ }^{1,4}$ carbides, $^{5-8}$ diborides, ${ }^{3,9-11}$ and disilicides, ${ }^{12-14}$ have been extensively investigated due to their high-melting point (>3273 K), excellent chemical stability at high temperature, and high hardness. Among these HECs, high-entropy disilicides are considered as the most promising high-temperature candidate material in that their individual components possess high melting points, high-temperature mechanical property retention, and excellent oxidation resistance. ${ }^{14}$ The fabrication of the high-entropy powders is critical for achieving the application of high-entropy disilicides. Up to now, limited research focused mainly on the fabrication of transition metal disilicide high-entropy bulk materials, ${ }^{12,13}$ whereas the synthesis of high-entropy disilicide (HES) powders was rarely reported.

In this communication, the high-entropy disilicides (HES) nanopowders, i.e., $\left(\mathrm{Nb}_{0.25} \mathrm{Ta}_{0.25} \mathrm{Hf}_{0.25} \mathrm{Ti}_{0.25}\right) \mathrm{Si}_{2}$, are fabricated by molten salt-assisted magnesium thermal reduction method. The influence of $\mathrm{Mg}$ contents on purity of HES powders was firstly studied and then the phase compositions, microstructure and compositional uniformity of the as-synthesized powders were investigated in detail, as well as the formation mechanism.

\section{Experimental Procedure}

The commercially transition metal oxides $\mathrm{Nb}_{2} \mathrm{O}_{5}, \mathrm{Ta}_{2} \mathrm{O}_{5}, \mathrm{TiO}_{2}, \mathrm{HfO}_{2}$ (99.9\% purity, particle size: 1-3 $\mu \mathrm{m}$, Shanghai Chao Wei Nanotechnology Co. Ltd), $\mathrm{SiO}_{2}$ (99\% purity, particle size: 1-3 $\mu \mathrm{m}$, Shanghai Chao Wei Nanotechnology Co. Ltd), Mg powders (99\% purity, particle size: 1-3 $\mu \mathrm{m}$, Shanghai Aladdin Biochemical Technology Co. Ltd), $\mathrm{NaCl}$ and KCl (99.5\% purity, Shanghai Aladdin Biochemical Technology Co. Ltd) were utilized to synthesize the HES nanopowders. The detailed fabrication process described as following: (1) $\mathrm{MeO}_{\mathrm{x}}(\mathrm{Me}=\mathrm{Nb}, \mathrm{Ta}, \mathrm{Ti}, \mathrm{Hf})$ powders were mixed with the equal molar fraction of metal 
atoms, $200 \mathrm{~mol} \%$ excess amount $\mathrm{SiO}_{2}$ powders, and $250 \mathrm{~mol} \%$ (350 mol\% and $400 \mathrm{~mol} \%$ ) excess amount $\mathrm{Mg}$ powders. The weigh ratios of mixture molten salt (the molar ration of $\mathrm{NaCl} / \mathrm{KCl}$ was $1: 1$, melting point at $\sim 737 \mathrm{~K}) /\left(\mathrm{MeO}_{\mathrm{X}}+\mathrm{Mg}+\mathrm{SiO}_{2}\right)$ were 10:1 according to previous work. ${ }^{8}$ Then, the mixture powders were heated to $1073 \mathrm{~K}$ for $6 \mathrm{~h}$ in a horizontal alumina tube furnace under a steady flow of Ar with a heating rate of $4 \mathrm{~K} / \mathrm{min}$. After that, the products were washed by deionized water at $363 \mathrm{~K}$ to remove the residual salts, followed by washing with $0.5 \mathrm{~mol} / \mathrm{L} \mathrm{HCl}$ solution to remove $\mathrm{MgO}$ byproduct. Finally, the products were washed by deionized water and dried at $363 \mathrm{~K}$.

The phase compositions, microstructure, and compositional uniformity of the as-synthesized powders were investigated by X-ray diffraction (XRD, X'pert PRO; PANalytical, Almelo, Netherlands), scanning electron microscopy (SEM, Supra-55; Zeiss, Oberkochen, Germany) equipped with energy dispersive spectroscopy (EDS), and transmission electron microscopy (TEM, Tecnai F30G2; FEI, Eindhoven, Netherlands) equipped with EDS.

\section{Results And Discussion}

The XRD patterns of the as-obtained samples were shown in Fig. 1. As shown in XRD pattern, when the molar ration of $\mathrm{Mg}$ content at $250 \mathrm{~mol} \%$, the primary phase was $\left(\mathrm{Ta}_{0.25} \mathrm{Hf}_{0.25} \mathrm{Ti}_{0.25} \mathrm{Nb}_{0.25}\right) \mathrm{Si}_{2}$ and the impurities, including $\mathrm{HfO}_{2}$ and $\mathrm{Nb}$, were also detected. Theoretically, the formation of $\mathrm{HfO}_{2}$ phase may lack of reducing agent, i.e., Mg powders (melting point $\sim 848 \mathrm{~K}$ ) because of the severe volatilization at such high temperature. To future address this issue, the molar ration of $\mathrm{Mg}$ content increased to $350 \mathrm{~mol} \%$ and $400 \mathrm{~mol} \%$; the XRD patterns were also shown in Fig. 1. Unexpected, the $\mathrm{HfO}_{2}$ still existed in the as-obtained powders and led to increase the $\mathrm{Nb}$ phase with increase the molar ration of $\mathrm{Mg}$. Thus, the $\mathrm{Mg}$ content is not a major factor affecting the purity of HES powders.

SEM images of the as-obtained HES nanopowders shown that they were all composed of many nanoparticles in the range of several nanometers to hundreds of nanometers (Fig. 2). Figure $2 b$ shows the EDS maps of four main transition metal elements in the as-synthesized powders at micrometer scale. Obviously, the distribution of elements is highly uniform without any aggregation or segregation. This indicates that the as-synthesized HES powders have highly compositional uniformity at microscale.

Figure 3a presents a typical TEM image of the as-obtained HES nanopowders. It obviously indicates that HES nanopowders consist of numerous individual nanoparticles. Figure $3 \mathrm{~b}$ shows a typical highresolution transmission electron microscopy image (HRTEM). It clearly exhibits a periodic lattice structure with a set of fringes with the d-space of $0.409 \mathrm{~nm}$, corresponding to the $\{100\}$ plane of HES phase, which is in good agreement with the calculation value $(0.414 \mathrm{~nm})$ from XRD pattern. Meanwhile, it should be noted that there is an amorphous layer with the thickness of 2-4 nm coated on surface, which may be due to the presence of amorphous $\mathrm{SiO}_{2}$. The selected area electron diffraction (SAED) pattern along with zone axis of [ indicates that the as-synthesized powders possess a single hexagonal structure. In addition, the average particle size of HES was confirmed to $\sim 55 \mathrm{~nm}$ according to 50 individual nanoparticles (Fig. 4). The scanning transmission electron microscopy (STEM) image and the 
corresponding EDS elemental maps (Fig. 4) of the as-synthesized HES nanopowders demonstrate that the distribution of three metal elements and $\mathrm{Si}$ element is basically uniform except Ti element aggregation at nanoscale.

Figure 5 demonstrates the possible formation mechanism of the as-synthesized HES powders via molten salt-assisted magnesium thermal reduction. It is generally accepted that the mixed salts firstly melted into the liquid during heat process (Fig. 5a). After that, four metal oxides, $\mathrm{SiO}_{2}$ and $\mathrm{Mg}$ gradually dissolved into the mixed molten salts (Fig. 5 b) by the following reactions: ${ }^{8}$

$$
\begin{gathered}
\mathrm{Mg} \rightarrow \mathrm{Mg}^{2+}+2 e^{-} \\
\mathrm{SiO}_{2} \rightarrow \mathrm{Si}^{4+}+2 \mathrm{O}^{2-} \\
\mathrm{MO}_{x} \rightarrow \mathrm{M}^{2 x+}+x \mathrm{O}^{2-}
\end{gathered}
$$

\section{$(4+x) M g^{2+}+2(4+x) e^{-}+M^{2 x+}+2 S i^{4+}+(4+x) O^{2-}$ \\ $\rightarrow \mathrm{HES}+(4+x) M g O$}

During this process, $\mathrm{Mg}$ gradually dissociated to mobile cations $\left(\mathrm{Mg}^{2+}\right)$ and delocalized electrons $\left(e^{-}\right)$in $\mathrm{NaCl} / \mathrm{KCl}$ molten salts according to Eq. 1. Meanwhile, four metal oxides and $\mathrm{SiO}_{2}$ also dissolved in $\mathrm{NaCl} / \mathrm{KCl}$ molten salts to produce mobile cations and anions. It has been reported that the molten salt synthesis method involves two mechanisms, including template formation and dissolutionprecipitation. According to the similarity-intermiscibility theory, ${ }^{15}$ it is considered that $\mathrm{SiO}_{2}$ is an atomic crystal; while the bond between four metal oxides and $\mathrm{NaCl} / \mathrm{KCl}$ is mainly ionic. The solubility of $\mathrm{SiO}_{2}$ in $\mathrm{NaCl} / \mathrm{KCl}$ molten salts is much less than that of four metal oxides. ${ }^{16}$ In this content, four transition metal cations would move to silicon ion surface and capture free electrons around it to generate HES molecules in molten salt according to Eq. 4 (Fig. 5c). Thus, it can be deduced that the formation mechanism in this work is mainly template formation mechanism. As the reaction proceeded, when the concentration of the generated HES molecules in molten salt medium reached the supersaturation condition, they could precipitate from the molten salt to nucleate and grow. Finally, numerous HES nanoparticles are obtained after removing the remnant reactants (Fig. $5 \mathrm{~d}$ ).

\section{Conclusion}

In summary, the high-entropy disilicide nanopowders, i.e., $\left(\mathrm{Ta}_{0.25} \mathrm{Hf}_{0.25} \mathrm{Ti}_{0.25} \mathrm{Nb}_{0.25}\right) \mathrm{Si}_{2}$, have been successfully fabricated by molten salt assisted magnesium thermal reduction for the first time. The asobtained nanopowders possessed a single hexagonal structure ( $\mathrm{TaSi}_{2}$-type) and high compositional uniformity at microscale, but shown Ti element aggregation at nanoscale. In addition, the template formation mechanism was proposed to explain the formation of HSE powders. 


\section{Declarations}

\section{Acknowledgements}

This work was supported National Natural Science Foundation of Guangdong Province (No. 2019A1515011002), and Foundation for the National Defense Key Laboratory (No. 6142907180302).

\section{References}

1. Rost CM, Sachet E, Borman T, Moballegh A, Dickey EC, Hou D et al (2015) Entropy-stabilized oxides. Nat Commun 6:8485

2. Ye B, Wen T, Nguyen M, Hao L, Wang C, Chu Y (2019) First-principles study, fabrication and characterization of $\left(\mathrm{Zr}_{0.25} \mathrm{Nb}_{0.25} \mathrm{Ti}_{0.25} \mathrm{~V}_{0.25}\right) \mathrm{C}$ high-entropy ceramics. Acta Mater 170:15-23

3. Gild J, Zhao Y, Harrington T, Jiang S, Hu T, Quinn MC et al (2016) High-entropy metal diborides: a new class of high-entropy materials and a new type of ultrahigh temperature ceramics. Sci Rep 6:37946

4. Zhao ZF, Xiang HM, Dai FZ, Peng ZJ, Zhou YC (2019) $\left(\mathrm{La}_{0.2} \mathrm{Ce}_{0.2} \mathrm{Nb}_{0.2} \mathrm{Sm}_{0.2} \mathrm{Eu}_{0.2}\right)_{2} \mathrm{Zr}_{2} \mathrm{O}_{7}$ : A novel high-entropy ceramic with low thermal conductivity and sluggish grain growth rate. J Mater Sci Technol 35:2647-2651

5. Wang F, Zhang $X$, Yan XL, Lu YF, Nastasi M, Chen $Y$ et al., The effect of submicron grain size on thermal stability and mechanical properties of high-entropy carbides ceramics. J Am Ceram Soc. Doi.org/10.1111/jace.17103

6. Feng L, Fahrenholtz W, Hilmas G (2019) Low-temperature sintering of single-phase, high-entropy carbide ceramics. J Am Ceram Soc 102(12):7217-7244

7. Du B, Liu HH, Chu YH (2020) Fabrication and characterization of polymer-derived high-entropy carbide ceramic powders. J Am Ceram Soc. DOI:10.1111/jace.17134

8. Ning S, Wen T, Ye B, Chu Y (2020) Low-temperature molten salt synthesis of high-entropy carbide powders. J Am Ceram Soc 103(3):2244-2251

9. Liu D, Liu HH, Ning SS, Ye BL, Chu YH (2019) Synthesis of high-purity high-entropy metal diboride powders by boro/carbothermal reduction. J Am Ceram Soc 102(12):7071-7076

10. Gu JF, Zou J, Sun SK, Wang H, Yu SY, Zhang JY et al (2019) Dense and pure high-entropy metal diboride ceramics sintered from self-synthesized powders via boro/carbothermal reduction approach. Sci China Mater 62:1-12

11. Zhang Y, Guo WM, Jiang ZB, Zhu QQ, Sun SK, You Y et al (2019) Dense high-entropy boride ceramics with ultra-high hardness. Scripta Mater 164:135-139

12. Gild J, Braun J, Kaufmann K, Marin E, Harrington T, Hopkins P et al (2019) A high-entropy silicide: $\left(\mathrm{Mo}_{0.2} \mathrm{Nb}_{0.2} \mathrm{Ta}_{0.2} \mathrm{Ti}_{0.2} \mathrm{~W}_{0.2}\right) \mathrm{Si}_{2}$. J Materiomics 5(3):337-343

13. Qin Y, Liu JX, Li F, Wei XF, Wu HZ, Zhang GJ (2019) A high entropy silicide by reactive spark plasma. J Adv Ceram 8:148-152 
14. Wen TQ, Liu HH, Ye BL, Liu D, Chu YH (2020) High-entropy alumino-silicides: a novel class of highentropy ceramics. Sci China Mater 63(2):300-306

15. Askeland DR, Fulay PP (2009) Essentials of materials science and engineering. Cengage Learning, Stanford

16. Gao FM, He JL, Wu ED, Liu SM, Yu DL, Li DC et al (2003) Hardness of covalent crystals. Phys Rev Lett 91(1):015502

\section{Figures}

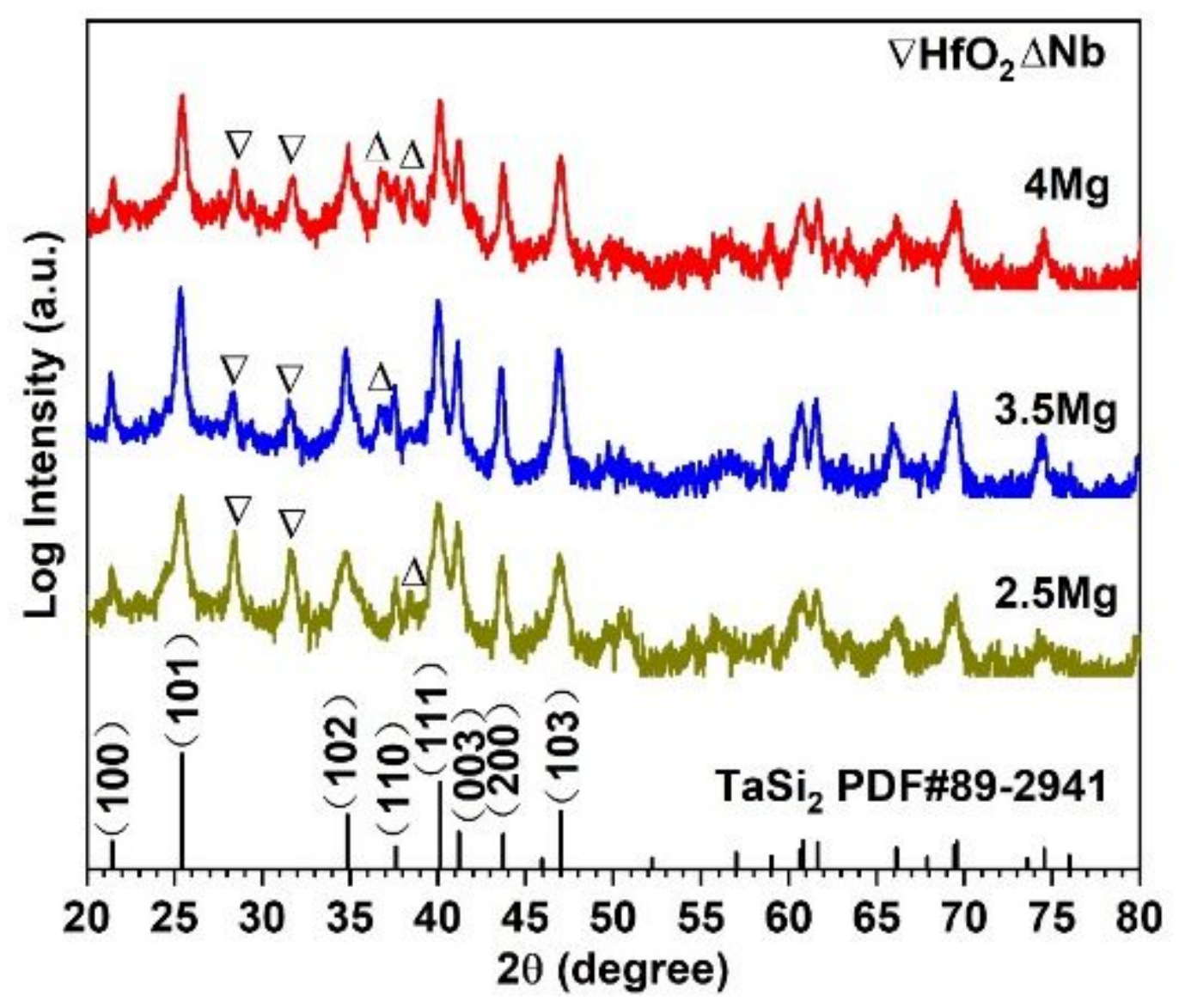

Figure 1

XRD patterns of the as-synthesized HES powders 


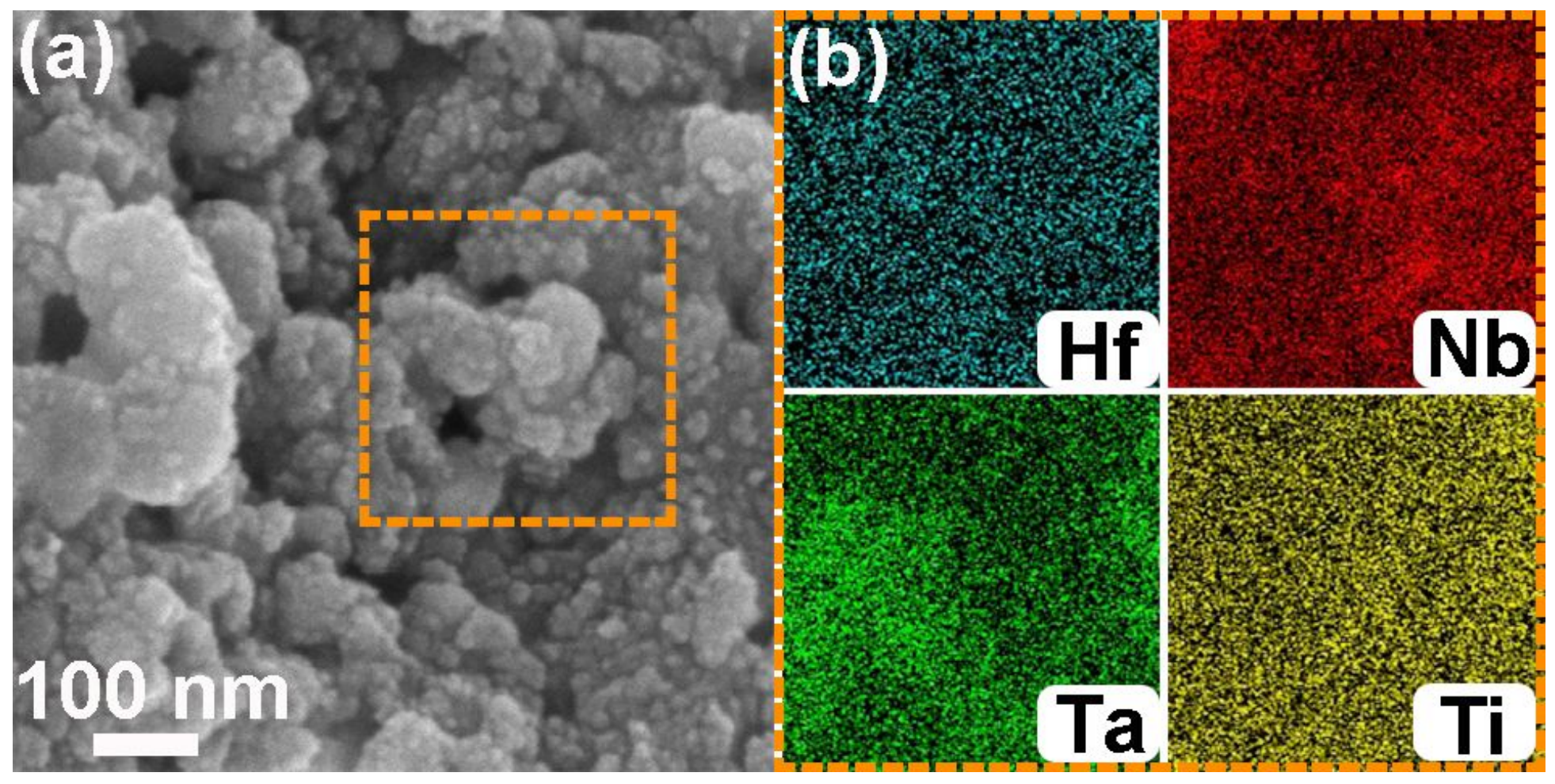

Figure 2

SEM characterizations of the as-synthesized HES powders (a) SEM image; (b) the corresponding EDS compositional maps (labeled by an orange square in Figure 1a)
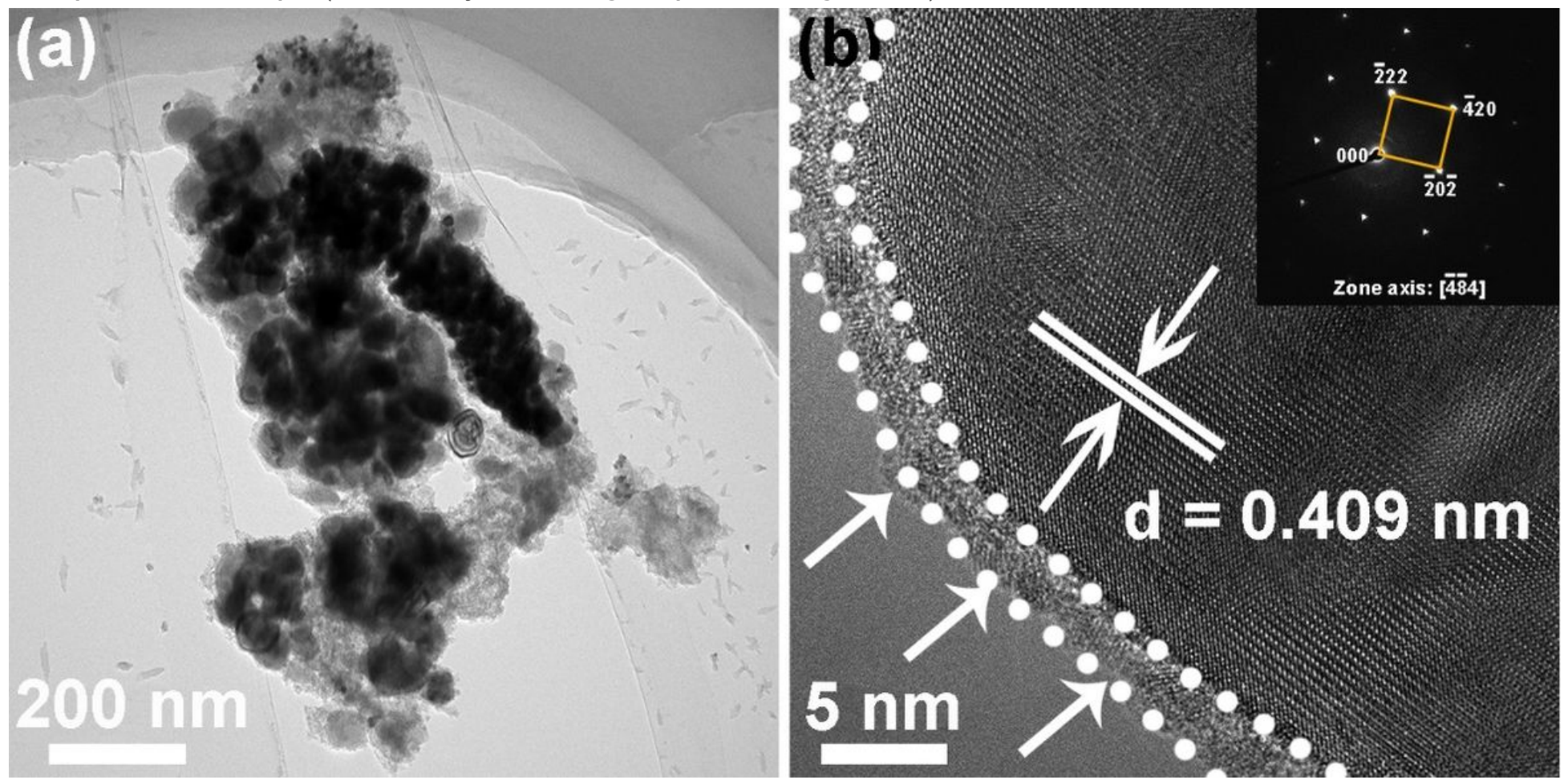

Figure 3

TEM analysis of the as-synthesized HES nanopowders (a) TEM image; (b) HRTEM image (insert shown the SAED pattern) 
(a)

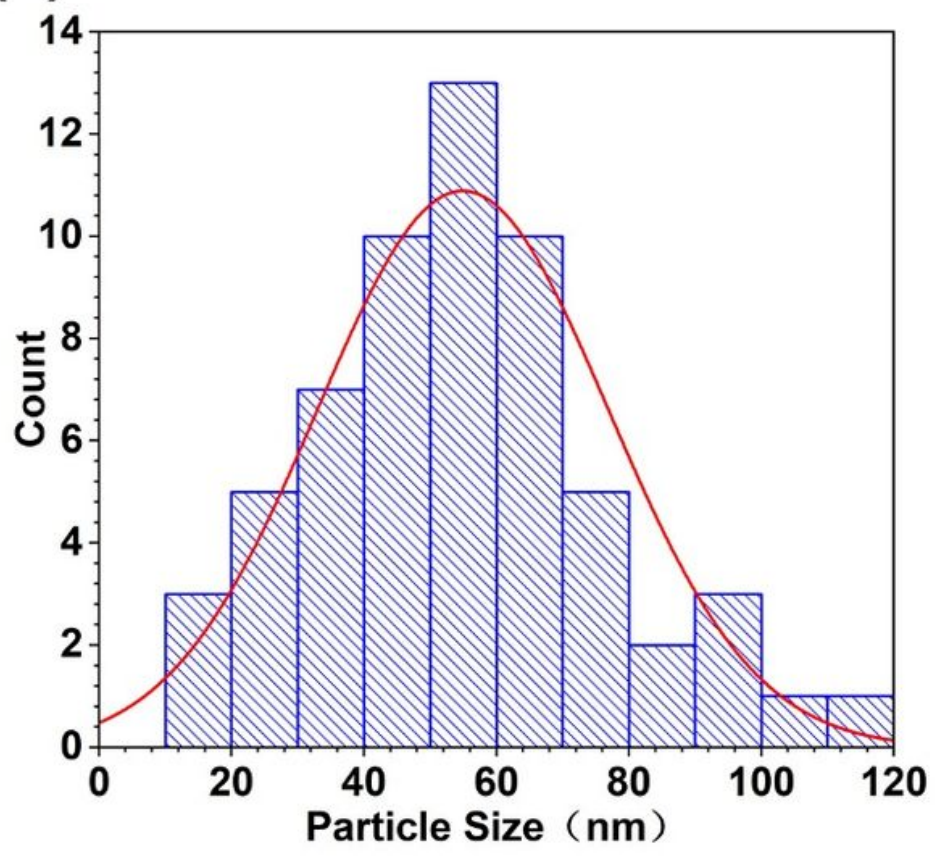

\section{(b)}

\section{$200 \mathrm{~nm}$}
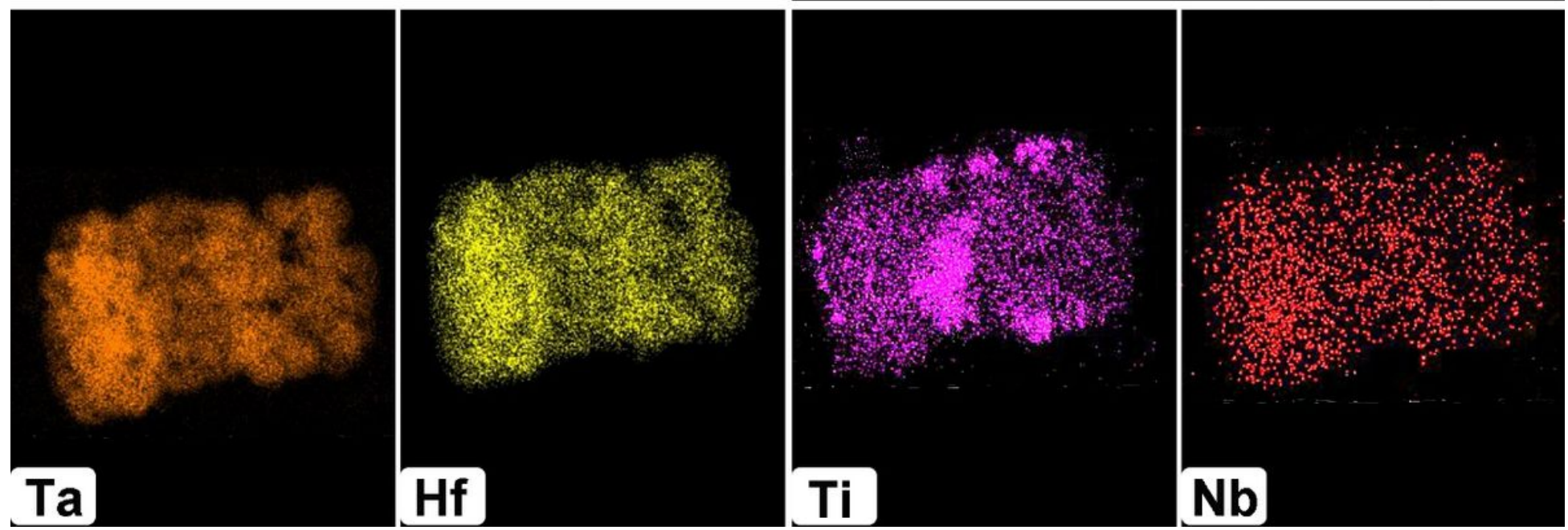

Figure 4

TEM analysis of the as-synthesized HES nanopowders: (a) histogram of the particle sizes with a Gaussian fitting to the data. The Gaussian peak is centered at $55 \mathrm{~nm}$; (b) STEM image and the corresponding EDS elemental maps 


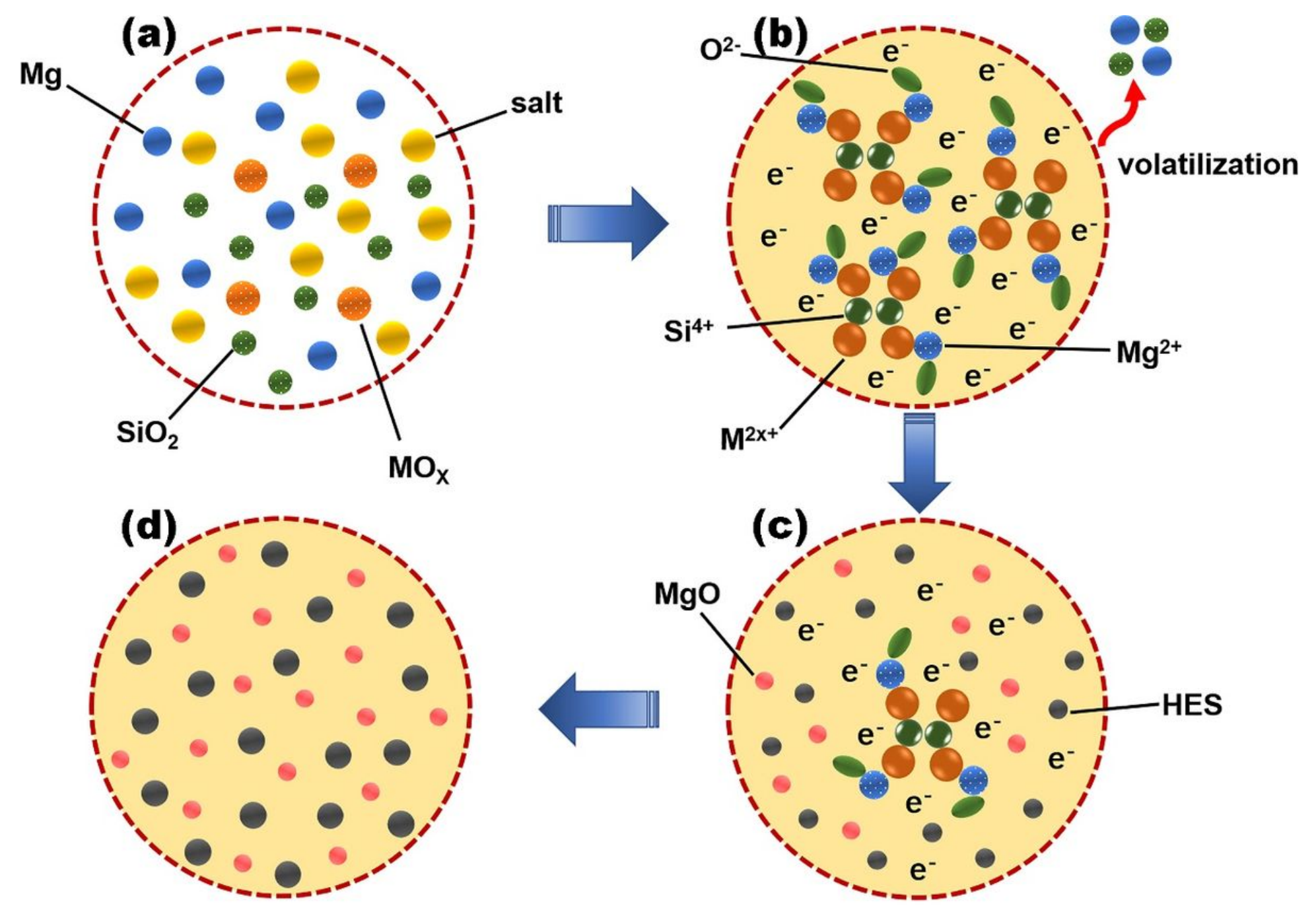

Figure 5

Schematic diagram of the possible formation process for HES nanoparticles 